\title{
P122 Unrealistic Pulse Pressures from Calibrated Tonometric Waveforms
}

\author{
Koen Reesink ${ }^{1, *}$, Louise Marais ${ }^{2}$, Pierre Boutouyrie ${ }^{2}$ \\ ${ }^{1}$ CARIM School of Cardiovascular Diseases, Maastricht University Medical Center, Maastricht, NL \\ ${ }^{2}$ INSERM, Paris, France
}

\begin{abstract}
Background: Calibration of carotid artery tonometry waveforms needs to account for pulse pressure augmentation [1]. Due to its location nearer to the heart, carotid pulse pressure (carPP) is about $10 \mathrm{mmHg}$ lower than brachial (brachPP) [1]. In practice, perfect applanation is hard to achieve with tonometry and thus tracking-errors may occur, leading to an exaggerated fall in the diastolic limb of the tonometry waveform. Consequently, the mean-to-diastolic difference of the waveform is underestimated, leading to an overestimation of carPP after calibration. We assessed the relationship between brachPP and carPP in 100 subjects, aged $18-80$ yrs.

Methods and Results: Brachial pressure (Omron) and carotid tonometry (Sphygmocor) measurements were performed by a single, highly skilled vascular technician, according to Artery Society methodological standards. CarPP was $5 \pm 13 \mathrm{mmHg}$ lower than brachPP. Bland-Altman analysis revealed a positive trend $(r=0.695, p<0.0001)$ between the carPP-brachPP difference and their mean, with carPP being $20 \mathrm{mmHg}$ lower than brachPP at a mean $40 \mathrm{mmHg}$ which extended to carPP being $20 \mathrm{mmHg}$ higher(!) than brachPP at a mean $80 \mathrm{mmHg}$. Accordingly, $30 \%$ of the study population showed unphysiological carPP, indicating an unrealistic i.e. reverse amplification. Even with a tolerance of $+5 \mathrm{mmHg}$, still $20 \%$ of cases showed unrealistic values.

Conclusion: In practice, calibrated carotid tonometry waveforms may yield unphysiological overestimations of carotid pulse pressure. This finding implies (potential) estimation errors in regression model studies on central pressure and local stiffness, suggesting brachial pulse pressure would be a valid compromise.
\end{abstract}

\section{REFERENCE}

[1] Kelly R, Fitchett D. Noninvasive determination of aortic input impedance and external left ventricular power output: a validation and repeatability study of a new technique. J Am Coll Cardiol 1992;20:952-63.

(c) 2019 Association for Research into Arterial Structure and Physiology. Publishing services by Atlantis Press International B.V. This is an open access article distributed under the CC BY-NC 4.0 license (http://creativecommons.org/licenses/by-nc/4.0/). 